\title{
Differential Games of Pursung in the Systems with Distributed Parameters and Geometrical Restrictions
}

\author{
M. Sh. Mamatov, E. B. Tashmanov, H. N. Alimov \\ Department “Geometry”, National University of Uzbekistan Named After M. Ulugbek, Tashkent, Uzbekistan \\ Email: mamatovmsh@mail.ru
}

Received 2013

\begin{abstract}
A problem of pursuit in the controlled systems of elliptic type without mixed derivatives with variable coefficients was considered. The model of the considered system is described by partial differential equations. The players (opponents) control parameters occur on the right-hand side of the equation and are subjected to various constraints. The first player's goal is to bring the system from one state into another desired state; the second player's goal is to prevent this from happening. We represent new sufficient conditions for bringing the system from one state into another. The finite-difference method is used to solve this problem.
\end{abstract}

Keywords: Pursuit; Pursuer; Evader; Terminal Set; Pursuit Control; Evasion control

\section{Introduction}

Some problem formulations in the theory of differential games may be illustrated by motion of two controlled objects, pursuer and evader. Let in the course of motion the objects continuously observe each other and at each time instant correct their motions depending on the information about the adversary. Depending on the pursuer's aim, the problem of pursuit is then formulated as follows: using the information about the evader, at each time instant $t$ select a control such that coincidence of the objects' spatial coordinates is reached as soon as possible.

The majority of studies consider the case where behavior of the lumped-parameter model described by a system of ordinary differential equations. This scheme encompasses many problems of differential games arising in diverse filed of the natural sciences. The mathematical issues of the differential games describing the lumped-parameter systems were developed in detail.

In many applications, however, the lumped-parameter models describe phenomena inadequately. It often turns out that a system which is optimal in the sense of a simplified model does not use the additional designed-in potentialities of control. The distributed-parameter models obeying the differential equations with partial derivatives offer a better, more adequate description. Use of these equations also gives rise to various game problems of which one is the subject matter of the present paper. It focuses only on the problem of pursuit. Therefore, we make an assumption about the nature of information for this problem.

\section{Formulation of the Problem}

The operated distributed system described by the elliptic equations (see, for example, [1,2]) is considered

$$
\begin{gathered}
a(x, y) \partial^{2} z / \partial x^{2}+b(x, y) \partial^{2} z / \partial y^{2}=f(u(x, y), v(x, y)), \\
\partial z / \partial \xi+\alpha(x, y) z=\varphi(x, y), \quad(x, y) \in \Gamma
\end{gathered}
$$

where $z=z(x, y)$ - unknown function, $a(x, y), b(x, y)$ - continuous functions in $\Omega=\{(x, y): 0<x<1,0<y<1\}$ with border $\Gamma, \varphi(x, y)$ - smooth function on $\Gamma, \xi$ - external normal. It is supposed that there is a positive constant $v$ such that for any $(x, y) \in \Omega$ the inequality, $b(x, y) \geq v, u=u(x, y), \quad v=v(x, y)$ - operating functions is executed from a class $L_{2}(\Omega)$. The first (pursuing) player, (pursued or escaping) the player, $u \in \bar{P}, v \in \bar{Q}$, $\bar{P}$ and $\bar{Q}$ - nonempty compacts in $R^{1}$ disposes of function $v(x, y)$ second of function $u(x, y)$. The terminal set $\bar{M}_{1} \subset R^{1}$ s allocated.

Definition 1. In a task (1) it is possible $\varepsilon$ - completion of $(\varepsilon>0)$ prosecutions from "boundary" situation $\phi(\cdot, \cdot)$, if exist function $u(v, x, y) \in \bar{P}, v \in \bar{Q}$, $(x, y) \in \Omega$, such that for any function $v_{0}(x, y) \in \bar{Q}$, $(x, y) \in \Omega$ the solution $z_{0}(x, t)$ of a task (1) where $u=u\left(v_{0}(x, y), x, y\right), \quad v=v_{0}(x, y)$, gets on a set $\varepsilon I+\bar{M}_{1}$, at some $(\tilde{x}, \tilde{y}), \quad(\tilde{x}, \tilde{y}) \in \Omega: z_{0}(\tilde{x}, \tilde{y}) \in \varepsilon I+\bar{M}_{1}$ where $I=(-1,1)$.

Decompose the Euclidean space $R^{2}$ of variables $(x, y)$ by the planes $x_{i}=i h, h=1 / r, i=0,1, \ldots$, and $y_{j}=j l, \quad l=1 / \theta, \quad j=0,1,2, \ldots, \quad$ into parallelepipeds 
$\Omega_{(i, j)}=\left\{(x, y): i h<x_{i}<(i+1) h, j l<y<(j+1) l\right\}, \quad r \quad$ and $\theta$ being some natural numbers. The points $\left(x_{i}, y_{j}\right)$ belonging to a set $\Omega$ are the nodes of the grid $\Omega_{h l}$. Each node has its neighbors. If all these neighbor nodes also belong to the grid $\Omega_{h l}$, then the node $\left(x_{i}, y_{j}\right)=$ $(i h, j l)$ is referred to as "internal", otherwise, $\left(x_{i}, y_{j}\right)$ is called the "boundary" node. The set of all boundary nodes is called as border of net area and is designated through $\Gamma_{h}$.

Replace the internal nodes of the derivatives (1) differential second-order accuracy of approximation ratios with formulas

$$
\begin{aligned}
& \partial z / \partial x\left(x_{i}, y_{j}\right)=\left(z\left(x_{i+1}, y_{j}\right)-z\left(x_{i-1}, y_{j}\right)\right) / 2 h+O(h)^{2}, \\
& \partial z / \partial y\left(x_{i}, y_{j}\right)=\left(z\left(x_{i}, y_{j+1}\right)-z\left(x_{i}, y_{j-1}\right)\right) / 2 l+O(l)^{2}, \\
& \partial^{2} z / \partial x^{2}\left(x_{i}, y_{j}\right)=\left(z\left(x_{i+1}, y_{j}\right)-2 z\left(x_{i}, y_{j}\right)+z\left(x_{i-1}, y_{j}\right)\right) / h^{2}+O(h)^{2}, \\
& \partial^{2} z / \partial y^{2}\left(x_{i}, y_{j}\right)=\left(z\left(x_{i}, y_{j+1}\right)-2 z\left(x_{i}, y_{j}\right)+z\left(x_{i}, y_{j-1}\right)\right) / l^{2}+O(l)^{2},
\end{aligned}
$$

Substituting these ratios in (1), having rejected an error of approximation of derivatives, we will receive the differential equations for unknown $z_{i, j}$

$$
\begin{gathered}
a_{i, j}\left(z_{i+1, j}-2 z_{i, j}+z_{i-1, j}\right) / h^{2}+b_{i, j}\left(z_{i, j+1}-2 z_{i, j}+z_{i, j-1}\right) / l^{2}=f_{i j}, \\
i=0,1, \ldots, r-1 ; \quad j=0,1, \ldots, \theta-1 .
\end{gathered}
$$

where the following designations of values of coefficients and the right part in a hub $\left(x_{i}, y_{j}\right): a_{i, j}, b_{i, j}, c_{i, j}, d_{i, j}$, $g_{i, j}, f_{i, j}$, for example are entered

$$
f_{i, j}=f\left(u\left(x_{i}, y_{j}\right), v\left(x_{i}, y_{j}\right)\right), \quad\left(x_{i}, y_{j}\right) \in \Omega_{h l}
$$

Ratios (2) contains except unknown $z_{i, j}$ in internal nodes also unknown $z_{i, j}$ on border of net area. For boundary nodes we will write down a ratio

$$
\begin{aligned}
& \left(z_{1, j}-z_{0, j}\right) / h+\alpha_{0, j}\left(z_{0, j}+z_{1, j}\right) / 2=\varphi_{0, j}, \quad(j=0,1,2, \ldots, \theta-1) \\
& \left(z_{r, j}-z_{r-1, j}\right) / h+\alpha_{r, j}\left(z_{r-1, j}+z_{r, j}\right) / 2=\varphi_{r, j}, \\
& \left(z_{i, 1}-z_{i, 0}\right) / l+\alpha_{i, 0}\left(z_{i, 1}+z_{i, 0}\right) / 2=\varphi_{i, 0}, \quad(i=0,1,2, \ldots, r-1) \\
& \left(z_{i, \theta}-z_{i, \theta-1}\right) / l+\alpha_{i, \theta}\left(z_{i, \theta}+z_{i, \theta-1}\right) / 2=\varphi_{i, \theta}
\end{aligned}
$$

Thus, we will receive system of $r \theta+2(r+\theta)$ equations with the same number of unknown $z_{i, j}$.

Using boundary conditions (3), we will express $z_{i, 1}$, $z_{i, \theta}$ through $z_{i, 0}, z_{i, \theta-1}$. Let's have

$$
\begin{aligned}
& z_{i, 1}=\left(2-l \alpha_{i, 0}\right) /\left(2+l \alpha_{i, 0}\right) z_{i, 0}+2 l \varphi_{i, 0}\left(2+l \alpha_{i, 0}\right) \\
& z_{i, \theta}=\left(2-l \alpha_{i, \theta}\right) /\left(2+l \alpha_{i, \theta}\right) z_{i, \theta-1}+2 l \varphi_{i, \theta}\left(2+l \alpha_{i, \theta}\right) .
\end{aligned}
$$

Using these ratios, we will exclude in system (3) unknown $z_{i, 1}, z_{i, \theta}$. If to enter designation $\gamma=h^{2} / l^{2}$, we will receive system

$$
\left.\begin{array}{c}
z_{i+1,0}-\left(2+2 \gamma-k_{i 0} \gamma\right) z_{i, 0}+\gamma z_{i, 1}+z_{i-1,0}=F_{i, 0}, \\
z_{i+1, j}+\gamma z_{i, j-1}-2(1+\gamma) z_{i, j}+\gamma z_{i, j+1}+z_{i-1, j}=F_{i, j} \\
(j=1,2, \ldots, \theta-2), \\
z_{i+1, \theta-1}+\gamma z_{i, \theta-2}-\left(2+2 \gamma-k_{i, \theta} \gamma\right) z_{i, \theta-1}+z_{i-1, \theta-1}=F_{i, \theta-1} \\
(i=0,1,2, \ldots, r-1),
\end{array}\right\}
$$

where

$$
\begin{aligned}
F_{i, 0}=h^{2} f_{i 0}-\gamma 2 l \varphi_{i, 0} /\left(2+l \alpha_{i, 0}\right) ; \quad F_{i j}=h^{2} f_{i, j}, \\
\\
(j=1,2, \ldots, \theta-2) ; \\
F_{i, \theta-1}=h^{2} f_{i, \theta-1}-\gamma 2 l \varphi_{i, \theta}\left(2+l \alpha_{i, \theta}\right) .
\end{aligned}
$$

This system can shortly be written down in a look

$$
z_{i+1}+A_{i} z_{i}+z_{i-1}=F_{i}, \quad(i=0,1,2, \ldots, r-1) .
$$

where

$$
\begin{aligned}
& z_{i}=\left(z_{i, 0}, z_{i, 1}, \ldots, z_{i, \theta-1}\right) ; \quad F_{i}=\left(F_{i, 0}, F_{i, 1}, \ldots, F_{i, \theta-1}\right), \\
& A_{i}=\left(\begin{array}{cccccc}
-2(1+\gamma)+k_{i, 0} \gamma & \gamma & 0 & \ldots & 0 & 0 \\
\gamma & -2(1+\gamma) & \gamma & \ldots & 0 & 0 \\
0 & \gamma & -2(1+\gamma) & \ldots & 0 & 0 \\
\ldots & \ldots & \ldots & \ldots \ldots & \ldots \\
0 & 0 & 0 & \ldots & \gamma & -2(1+\gamma)+k_{i, \theta} \gamma
\end{array}\right)
\end{aligned}
$$

Boundary conditions (3) and (5) can be copied in a look

$$
\begin{aligned}
z_{1, j} & =\left(2-h \alpha_{0, j}\right) / 2+h \alpha_{0, j} z_{0, j}+\left(2 h \varphi_{0, j}\right) /\left(2+h \alpha_{0, j}\right) \\
& =k_{0, j} z_{0, j}+y_{0, j}, \quad(j=0,1,2, \ldots, \theta-1) \\
z_{r-1, j} & =\left(2+h \alpha_{r, j}\right) /\left(2-h \alpha_{r, j}\right) z_{r, j}-\left(2 h \varphi_{r, j}\right) /\left(2-h \alpha_{r, j}\right) \\
& =k_{r, j} z_{r, j}+y_{r, j}, \quad(j=0,1,2, \ldots, \theta-1)
\end{aligned}
$$

where

$$
\begin{aligned}
& k_{0, j}=\left(2-h \alpha_{0, j}\right) /\left(2+h \alpha_{0, j}\right) ; y_{0, j}=\left(2 h \varphi_{0, j}\right) /\left(2+h \alpha_{0, j}\right) ; \\
& k_{r, j}=\left(2+h \alpha_{r, j}\right) /\left(2-h \alpha_{r, j}\right) ; \quad y_{r, j}=-\left(2 h \varphi_{r, j}\right) /\left(2-h \alpha_{r, j}\right) .
\end{aligned}
$$

Having put

$$
\begin{aligned}
& y_{0}=\left(y_{0,0}, y_{0,1}, \ldots, y_{0, \theta-1}\right) ; y_{r}=\left(y_{r, 0}, y_{r, 1}, \ldots, y_{r, \theta-1}\right) \text {. } \\
& X_{0}=\left(\begin{array}{cccccc}
k_{0,0} & 0 & 0 & \ldots & 0 & 0 \\
0 & k_{0,1} & 0 & \ldots & 0 & 0 \\
\ldots & \ldots & \ldots & \ldots & \ldots & \ldots \\
0 & 0 & 0 & \ldots & 0 & k_{0, \theta-1}
\end{array}\right) ; \\
& X_{1}=\left(\begin{array}{cccccc}
k_{r, 0} & 0 & 0 & \ldots & 0 & 0 \\
0 & k_{r, 1} & 0 & \ldots & 0 & 0 \\
\ldots & \ldots & \ldots & \ldots & \ldots & \ldots \\
0 & 0 & 0 & \ldots & 0 & k_{r, \theta-1}
\end{array}\right),
\end{aligned}
$$

it is possible to write down systems (9) in such look:

$$
\left.\begin{array}{c}
z_{1}=X_{0} z_{0}+y_{0}, \\
z_{r-1}=X_{1} z_{r}+y_{r} .
\end{array}\right\}
$$

Finally we have the following system of the equations:

$$
\begin{aligned}
z_{1} & =X_{0} z_{0}+y_{0}, \\
z_{i+1}+A_{i} z_{i}-z_{i-1} & =F_{i} \quad(i=1,2, \ldots, r-1) \\
z_{r-1} & =X_{1} z_{r}+y_{r} \text { 。 }
\end{aligned}
$$

Instead of game (13) we will consider more the general game described by system of the equations

$$
\begin{gathered}
C_{0} z_{0}-B_{0} z_{1}=f_{0}, \\
-A_{n} z_{n-1}+C_{n} z_{n}-B_{n} z_{n+1}=f_{n}\left(u_{n}, v_{n}\right), 1 \leq n \leq N-1, \\
-A_{N} z_{N-1}+C_{N} z_{N}=f_{N},
\end{gathered}
$$

where $z_{n} \in R^{m}, \quad n=\overline{0, N}, \quad A_{n}, C_{n}, B_{n}-m \times m$ constant square matrixes, $u_{n}, v_{n}$ - operating parameters, $u_{n}$ 
prosecution parameter, $v_{n}$ - beanie parameter, $u_{n} \in P_{n}$ $\subset R^{p}, v_{n} \in Q_{n} \subset R^{q}, P_{n}$ and $Q_{n}$ - nonempty sets; $f_{n}$ - the set function displaying $R^{p} \times R^{q}$ in $R^{m}$. Besides, in $R^{m}$ the terminal set is $M$ allocated.

Definition 2. We shall say that from "boundary" situation $\left(f_{0}, f_{N}\right)$ it is possible to complete pursuit for $N$ steps if from any sequence $\bar{v}_{1}, \bar{v}_{2}, \ldots, \bar{v}_{N-1}$ of the values of evasion controls it is possible to construct a sequence $\bar{u}_{1}, \bar{u}_{2}, \ldots, \bar{u}_{N-1}$ of values of the pursuit control values such that the solution $\left\{z_{0}, \bar{z}_{1}, \bar{z}_{2}, \ldots, \bar{z}_{N-1}, z_{N}\right\}$ of the equation

$$
\begin{gathered}
C_{0} z_{0}-B_{0} z_{1}=f_{0}, \\
-A_{n} z_{n-1}+C_{n} z_{n}-B_{n} z_{n+1}=f_{n}\left(\bar{u}_{n}, \bar{v}_{n}\right), 1 \leq n \leq N-1, \\
-A_{N} z_{N-1}+C_{N} z_{N}=f_{N} .
\end{gathered}
$$

Gets on $M: \bar{z}_{i} \in M$ for some $i$. Thus for finding of value $\bar{u}_{n}$ it is allowed to use values $\bar{v}_{n}$ and $\bar{z}_{n}$.

Note that the type of systems (14) is difference schemes for elliptic equations of second order with variable coefficients in any field of any number of dimensions [3-14].

Solution of problem (14) will be sought in the form

$$
z_{n}=\alpha_{n+1} z_{n+1}+\beta_{n+1}, n=N-1, N-2, \ldots, 0,
$$

where $\alpha_{n+1}$ - uncertain while a square matrix of the sizes $m \times m$, and $\beta_{n+1}-$ a vector of dimension $m$. From a formula (16) and the equations of system (14) for $1 \leq n \leq N-1$ there are recurrent ratios for calculation of matrixes $\alpha_{n}$ and vectors $\beta_{n}$. Really from a formula (16) $z_{n-1}=\alpha_{n} z_{n}+\beta_{n}$ substituting it in (14) we will receive

$$
\begin{gathered}
-A_{n}\left(\alpha_{n} z_{n}+\beta_{n}\right)+C_{n} z_{n}-B_{n} z_{n+1}=f_{n}\left(u_{n}, v_{n}\right), \\
1 \leq n \leq N-1 ; \\
\left(C_{n}-A_{n} \alpha_{n}\right) z_{n}=B_{n} z_{n+1}+f_{n}\left(u_{n}, v_{n}\right)+A_{n} \beta_{n} ; \\
z_{n}=\left(C_{n}-A_{n} \alpha_{n}\right)^{-1} B_{n} z_{n+1}+\left(C_{n}-A_{n} \alpha_{n}\right)^{-1}\left[f_{n}\left(u_{n}, v_{n}\right)+A_{n} \beta_{n}\right] .
\end{gathered}
$$

Equating now the right parts of the last and (16) equalities we will receive

$$
\begin{gathered}
\alpha_{n+1}=\left(C_{n}-A_{n} \alpha_{n}\right)^{-1} B_{n}, \quad n=1,2, \ldots, N-1 ; \\
\beta_{n+1}=\left(C_{n}-A_{n} \alpha_{n}\right)^{-1}\left[f_{n}\left(u_{n}, \cup_{n}\right)+A_{n} \beta_{n}\right], \\
n=1,2, \ldots, N .
\end{gathered}
$$

Further from (16) and the equations (14) for $n=0, N$, there are the initial values $\alpha_{1}, \beta_{1}$ and $z_{N}$, allowing beginning the account on recurrent ratios. From (14) and (16) for $n=0$ we will have

$$
z_{0}=C_{0}^{-1} B_{0} z_{1}+C_{0}^{-1} f_{0}, \quad z_{0}=\alpha_{1} z_{1}+\beta_{1},
$$

And, therefore

$$
\alpha_{1}=C_{0}^{-1} B_{0}, \quad \beta_{1}=C_{0}^{-1} f_{0} .
$$

In the same way for $n=N$ we have

$$
-A_{N}\left(\alpha_{N} z_{N}+\beta_{N}\right)+C_{N} z_{N}=f_{N}
$$

or

$$
z_{N}=\left(C_{N}-A_{N} \alpha_{N}\right)^{-1}\left(f_{N}+A_{N} \beta_{N}\right) .
$$

Uniting, we will write out final formulas

$$
\begin{gathered}
\alpha_{n+1}=\left(C_{n}-A_{n} \alpha_{n}\right)^{-1} B_{n}, \quad n=1,2, \ldots, N, \quad \alpha_{1}=C_{0}^{-1} B_{0} \\
\beta_{n+1}=\left(C_{n}-A_{n} \alpha_{n}\right)^{-1}\left(f_{n}\left(u_{n}, v_{n}\right)+A_{n} \beta_{n}\right), \\
n=1,2, \ldots, N-1 . \quad \beta_{1}=C_{0}^{-1} f_{0}, \\
z_{n}=\alpha_{n+1} z_{n+1}+\beta_{n+1}\left(u_{n}, v_{n}\right), \\
n=N-1, N-2, \ldots, 0, \quad z_{N}=\beta_{N+1}
\end{gathered}
$$

It is clear, that if in game (17), (18), (19) $z_{\bar{n}} \in M$ that in game (14) too game comes to the end. Therefore further instead of game (14) we will consider discrete game described by system of the equations (17), (18), (19).

Before giving determination of stability of algorithm (17), (18), (19), we will provide some data from linear algebra.

Let $A$ - any square matrix $m \times m$ and $\|x\|_{m}$ be norm of a vector in $R^{m}$, then the norm $A$ is defined by equality

$$
\|A\|=\sup _{x \neq 0}\|A x\|_{m} /\|x\|_{m} .
$$

For a case of Euclidean norms in $R^{m}$ we have $\|A\|=\sqrt{\rho}$, where $\rho$ - maximum on the module own value of a matrix $A \cdot A$.

Without the proof we will give the following known lemma (see [15]).

Lemma 1. Let for some matrix norm the square matrix meet a condition $\|A\| \leq q<1$. Then there is a matrix $(E+A)^{-1}$ and $\left\|(E+A)^{-1}\right\| \leq 1 /(1-q)$.

Let's say that the algorithm is steady if the assessment $\left\|\alpha_{j}\right\| \leq 1$ for $1 \leq j \leq N$ is carried out.

Lemma 2. If $C_{j}$ for $0 \leq j \leq N$ - no degenerate matrixes and $A_{j}$ and $B_{j}$ - nonzero matrixes for $1 \leq j \leq$ $N-1$ also are satisfied conditions

$$
\begin{gathered}
\left\|C_{0}^{-1} B_{0}\right\| \leq 1, \quad\left\|C_{N}^{-1} A_{N}\right\| \leq 1, \\
\left\|C_{j}^{-1} A_{j}\right\|+\left\|C_{j}^{-1} B_{j}\right\| \leq 1, \quad 1 \leq j \leq N-1 .
\end{gathered}
$$

And at least in one of inequalities the strict inequality takes place, there are return to the $C_{j}-A_{j} \alpha_{j}$. matrix and $\left\|\alpha_{j}\right\| \leq 1$, here $\alpha_{1}=C_{0}^{-1} B_{0}$,

$$
\alpha_{j+1}=\left(C_{j}-A_{j} \alpha_{j}\right)^{-1} B_{j}, \quad 1 \leq j \leq N-1 .
$$

Proof. $\left\|\alpha_{1}\right\|=\left\|C_{0}^{-1} B_{0}\right\| \leq 1$, suppose, that $\left\|\alpha_{j}\right\| \leq 1$ also we will show $\left\|\alpha_{j+1}\right\| \leq 1$. After a course the proof of this fact we will receive existence of a matrix $\left(C_{j}-A_{j} \alpha_{j}\right)^{-1}$. Really from conditions of a lemma we will have

$$
\left\|C_{j}^{-1} A_{j} \alpha_{j}\right\| \leq\left\|C_{j}^{-1} A_{j}\right\| *\left\|\alpha_{j}\right\| \leq\left\|C_{j}^{-1} A_{j}\right\| \leq 1-\left\|C_{j}^{-1} B_{j}\right\|<1 .
$$

As $C_{j}^{-1} A_{j} \alpha_{j}$ square matrix that owing to a lemma 1 there are return to $E-C_{j}^{-1} A_{j} \alpha_{j}$ and $C_{j}-A_{j} \alpha_{j}$ matrixes and $\left\|\left(E-C_{j}^{-1} A_{j} \alpha_{j}\right)\right\| \leq 1 /\left\|C_{j}^{-1} B_{j}\right\|$. From here and from (17) we will receive

$$
\begin{aligned}
& \left\|\alpha_{j+1}\right\| \leq\left\|\left(E-C_{j}^{-1} A_{j} \alpha_{j}\right)^{-1} C_{j}^{-1} B_{j}\right\| \\
& \leq\left\|\left(E-C_{j}^{-1} A_{j} \alpha_{j}\right)^{-1}\right\| \cdot\left\|C_{j}^{-1} B_{j}\right\| \leq 1 .
\end{aligned}
$$

The proof of the lemma is complete. 


\section{Main Results}

Everywhere further it is supposed that $M=M_{0}+M_{1}$, where $M_{0}$ - linear subspace $R^{m}, M_{1}$ - a subset a subspace, $L$ - orthogonal complement of $M_{0}$ in $R^{n}$. Denote $\Pi$ we will designate a matrix of orthogonal design from $R^{m}$ on $L$.

Let $W(0)=\{0\}$,

$$
\begin{gathered}
W(k)=\sum_{i=0}^{k-1} \bigcap_{v_{N-k+i} \in Q_{N-k+i}} \prod \alpha_{N-k} \ldots \alpha_{N-k+i} \beta_{N-k+i+1}\left(P_{N-k+i}, v_{N-k+i}\right) \\
W_{1}(k)=-\mathrm{M}_{1}+W(k), \quad 1 \leq k \leq N .
\end{gathered}
$$

Theorem 1. Let $\bar{N}$ be the smallest of the numbers $k$, such that

$$
-\prod \alpha_{N-k} \alpha_{N-k+1} \ldots \alpha_{N} z_{N} \in W_{1}(k) .
$$

Then from "boundary" situation $\left(f_{0}, f_{N}\right)$ it is possible to complete pursuit for $\bar{N}$ steps.

Let now $W_{2}(0)=-M_{1}$,

$$
\begin{aligned}
& W_{2}(1)=\bigcap_{v_{N-k-1} \in Q_{N-k-1}}\left[W_{2}(0)+\prod \beta_{N-k}\left(P_{N-k-1}, v_{N-k-1}\right)\right], \\
& W_{2}(k)=\bigcap_{v_{N-1} \in Q_{N-1}}\left[W_{2}(k-1)+\prod \alpha_{N-k} \alpha_{N-k+1} \ldots \alpha_{N-1} \beta_{N}\left(P_{N-1}, v_{N-1}\right)\right]
\end{aligned}
$$

Theorem 2. If $\bar{N}$ be smallest of those numbers $k$, for each of which takes place inclusion

$$
-\prod \alpha_{N-k} \alpha_{N-k+1}{ }^{\cdots} \alpha_{N^{z}} \in W_{2}(k)
$$

that of "boundary" situation $\left(f_{0}, f_{N}\right)$ it is possible to complete pursuit for $\bar{N}$ steps.

Let

$$
\gamma_{k}(\cdot)=\left\{\gamma_{0}, \gamma_{1}, \ldots, \gamma_{k-1}: \gamma_{i} \geq 0, \sum_{i=0}^{k-1} \gamma_{i}=1\right\}
$$

and

$$
\begin{aligned}
& W\left(\gamma_{k}(\cdot)\right) \\
&= \sum_{i=0}^{k-1} \bigcap_{v_{N-k+i} \in Q_{M-k+i}}\left[-\gamma_{i} M_{1}+\prod \alpha_{N-k} \ldots \alpha_{N-k+i} \beta_{N-k+i+1}\left(P_{N-k+i}, v_{N-k+i}\right)\right], \\
& 0 \leq k \leq N, \\
& W_{3}(0)=-M_{1}, \quad W_{3}(k)=\bigcup_{\gamma_{k}(\cdot)} W\left(\gamma_{k}(\cdot)\right), 0 \leq k \leq N .
\end{aligned}
$$

Theorem 3. If $M_{1}-$ a convex set and $\bar{N}$ be smallest of those numbers $k$. For each of which inclusion takes place

$$
-\prod \alpha_{N-k} \alpha_{N-k+1} \ldots \alpha_{N} z_{N} \in W_{3}(k) .
$$

That of "boundary" situation $\left(f_{0}, f_{N}\right)$ it is possible to complete pursuit for $\bar{N}$ steps.

It is easy to be convinced [15] that the solution of $z_{i, j}$ differential task (2) meets to the solution $z$ of an initial task (1), the following assessment of speed of convergence takes place

$$
\left\|(z)_{h l}-z_{i, j}\right\|_{\Phi_{h l}} \leq K_{1} h^{2}+K_{2} l^{2},
$$

where $(z)_{h l}$ - values of the exact decision a task (1) in grid functions, $\Phi_{h l}$ - spaces of net functions, $\|\cdot\|_{\Phi_{h l}}-$ is its norm and, $K_{1}$ and $K_{2}$ constants.

Theorem 4. Let in an inequality (26) $K_{1} h^{2}+K_{2} l^{2}<\varepsilon$, and in game (13) from a "boundary" situation $\left(f_{0}, f_{N}\right)=$ $\left(-y_{0},-y_{N}\right)$ completion of prosecution that is definitions 2 be possible. Then in game (1) from "boundary" situation $\partial z / \partial \xi+\alpha(x, y) z=\varphi(x, y), \quad(x, y) \in \Gamma$ it is possible to complete pursuit that are definitions 1 .

\section{Proof of Theorem}

Proof of Theorem 1. Let $\bar{v}_{1}, \bar{v}_{2}, \ldots, \bar{v}_{N-1}, \bar{v}_{i} \in Q_{i}$, $1 \leq i \leq N-1$ - any sequence. Instead of inclusion (21) we will consider other inclusion equivalent to it

$$
\begin{aligned}
& -\prod \alpha_{N-k} \alpha_{N-k+1} \ldots \alpha_{N} z_{N} \in W_{1}(k-1) \\
& +\bigcap_{v_{N-1} \in Q_{N-1}} \prod \alpha_{N-k} \alpha_{N-k+1} \ldots \alpha_{N-1} \beta_{N}\left(P_{N-1}, v_{N-1}\right)
\end{aligned}
$$

Means, exists $a_{N-1}$

$$
a_{N-1} \in \bigcap_{v_{N-1} \in Q_{N-1}} \prod \alpha_{N-k} \alpha_{N-k+1} \ldots \alpha_{N-1} \beta_{N}\left(P_{N-1}, v_{N-1}\right) .
$$

Such that

$$
-\prod \alpha_{N-k} \alpha_{N-k+1} \ldots \alpha_{N} z_{N} \in W_{1}(k-1)+a_{N-1} .
$$

Now control of the pursuing player $\bar{u}_{N-1}$, the relevant control of the escaping player $\bar{v}_{N-1}$, we will construct as the solution of the following control

$$
\prod \alpha_{N-k} \alpha_{N-k+1} \ldots \alpha_{N-1} \beta_{N}\left(u_{N-1}, \bar{v}_{N-1}\right)=a_{N-1} .
$$

It is clear, that the equation has the decision. From here owing to (27) we have

$$
\begin{aligned}
& -\prod \alpha_{N-k} \alpha_{N-k+1} \ldots \alpha_{N} z_{N} \\
& \in W_{1}(k-1)+\alpha_{N-k} \alpha_{N-k+1} \ldots \alpha_{N-1} \beta_{N}\left(\bar{u}_{N-1}, \bar{v}_{N-1}\right)
\end{aligned}
$$

We write down this inclusion in other look.

$$
-\prod \alpha_{N-k} \alpha_{N-k+1} \ldots \alpha_{N-1}\left[\alpha_{N} z_{N}+\beta_{N}\left(\bar{u}_{N-1}, \bar{v}_{N-1}\right)\right] \in W_{1}(k-1) .
$$

As a result from equalities (18) and (28) we will receive

$$
-\prod \alpha_{N-k} \alpha_{N-k+1} \ldots \alpha_{N-1} z_{N-1} \in W_{1}(k-1)
$$

Done above a reasoning allow us to construct on the set control $\bar{v}_{N-1}$ providing inclusion (29). If now the control $\bar{v}_{N-2}$ becomes known that, we above can receive in the stated way control $\bar{u}_{N-1}$ providing inclusion

$$
-\prod \alpha_{N-k} \alpha_{N-k+1} \ldots \alpha_{N-2} z_{N-2} \in W_{1}(k-2) \text {. }
$$

Repeating this process, further we can construct step by step control $\bar{u}_{i}$, proceeding from becoming known controls $\bar{v}_{i}$, therefore, that in any step inclusion takes place

$$
-\prod z_{N-k-1} \in W_{1}(0)=-\mathrm{M}_{1} .
$$

It means that 


$$
\prod z_{N-k-1} \in \mathrm{M}
$$

As we set out to prove.

Proof of Theorem 2. Let $\bar{v}_{1}, \bar{v}_{2}, \ldots, \bar{v}_{N-1}, \quad \bar{v}_{i} \in Q_{i}$, $1 \leq i \leq N-1$ - any sequence. For concrete $\bar{v}_{N-1}$ owing to (22) and (23) we will receive inclusion

$$
\begin{aligned}
& -\prod \alpha_{N-k} \alpha_{N-k+1} \ldots \alpha_{N} z_{N} \in W_{2}(k-1) \\
& +\prod \alpha_{N-k} \alpha_{N-k+1} \ldots \alpha_{N-1} \beta_{N}\left(P_{N-1}, \bar{v}_{N-1}\right)
\end{aligned}
$$

Now as $\bar{u}_{N-1}$ we take that element from $P_{N-1}$ for which inclusion (30) remained. Then we will receive

$$
\begin{aligned}
& -\prod \alpha_{N-k} \alpha_{N-k+1} \ldots \alpha_{N} z_{N} \in W_{2}(k-1) \\
& +\prod \alpha_{N-k} \alpha_{N-k+1} \ldots \alpha_{N-1} \beta_{N}\left(\bar{u}_{N-1}, \bar{v}_{N-1}\right)
\end{aligned}
$$

From this it follows that

$$
-\prod \alpha_{N-k} \alpha_{N-k+1} \ldots \alpha_{N-1}\left[\alpha_{N} z_{N}+\beta_{N}\left(\bar{u}_{N-1}, \bar{v}_{N-1}\right)\right] \in W_{2}(k-1) .
$$

And therefore, owing to (19) we have

$$
-\prod \alpha_{N-k} \alpha_{N-k+1} \ldots \alpha_{N-1} z_{N-1} \in W_{2}(k-1) .
$$

If now the control $\bar{v}_{N-1}$ becomes the stated way known that we above us can construct control $\bar{u}_{N-1}$ providing inclusion

$$
-\prod \alpha_{N-k} \alpha_{N-k+1} \ldots \alpha_{N-2} z_{N-2} \in W_{2}(k-2) .
$$

Further arguing similarly in any step we will receive

$$
-\prod z_{N-k-1} \in W_{2}(0)=-\mathrm{M}_{1},
$$

that is

$$
z_{N-k-1} \in \mathrm{M} .
$$

The theorem is proved completely.

Proof of Theorem 3. Instead of inclusion (25) meaning (24) we will consider inclusion equivalent to it

$$
-\prod \alpha_{N-k} \alpha_{N-k+1} \ldots \alpha_{N} z_{N} \in W(\bar{\gamma}(\cdot)) \text {. }
$$

Existence $\bar{\gamma}(\cdot)=\left\{\bar{\gamma}_{0}, \bar{\gamma}_{1}, \ldots, \bar{\gamma}_{k-1}, \bar{\gamma}_{i} \geq 0, \sum_{i=0}^{k-1} \bar{\gamma}_{i}=1\right\} \quad$ follows from (24). From here follows

$$
\begin{aligned}
& -\prod \alpha_{N-k} \alpha_{N-k+1} \ldots \alpha_{N} z_{N} \in \sum_{i=0}^{k-2} \bigcap_{v_{N-k+\in} \in Q_{N-k+i}} \\
& {\left[-\bar{\gamma}_{i} \mathrm{M}_{1}+\prod \alpha_{N-k} \ldots \alpha_{N-k+i} \beta_{N-k+i+1}\left(P_{N-k+i}, U_{N-k+i}\right)\right]} \\
& +\bigcap_{v_{N-1} \in Q_{N-1}}\left[-\bar{\gamma}_{k-1} \mathrm{M}_{1}+\prod \alpha_{N-k} \ldots \alpha_{N-1} \beta_{N}\left(P_{N-1}, v_{N-1}\right)\right] .
\end{aligned}
$$

Let now $\bar{v}_{1}, \bar{v}_{2}, \ldots, \bar{v}_{N-1}, \quad \bar{v}_{i} \in Q_{i}, \quad 1 \leq i \leq N-1$ - any sequence. Owing to (31) exists such $a_{N-1}$ that

$$
\begin{aligned}
& v_{N-1} \in \bigcap_{v_{N-1 \epsilon} \in Q_{N-1}}\left[-\bar{\gamma}_{k-1} \mathrm{M}_{1}+\prod \alpha_{N-k} \ldots \alpha_{N-1} \beta_{N}\left(P_{N-1}, v_{N-1}\right)\right], \\
& -\prod \alpha_{N-k} \alpha_{N-k+1} \ldots \alpha_{N} z_{N} \in \sum_{i=0}^{k-2} \bigcap_{v_{N-k+i} Q_{N-k+i}} \\
& {\left[-\bar{\gamma}_{i} \mathrm{M}_{1}+\prod \alpha_{N-k} \ldots \alpha_{N-k+i} \beta_{N-k+i+1}\left(P_{N-k+i}, v_{N-k+i}\right)\right]+a_{N-1}}
\end{aligned}
$$

Therefore, controls $\bar{u}_{N-1}$ we will construct as the solution of the following equation

$$
-\bar{\gamma}_{k-1} m_{1}+\Pi \alpha_{N-k} \ldots \alpha_{N-1} \beta_{N}\left(u_{N-1}, \bar{v}_{N-1}\right)=a_{N-1}, m_{1, k-1} \in \mathrm{M}_{1} .
$$

Further owing to (32) we have

$$
\begin{aligned}
& -\prod \alpha_{N-k} \alpha_{N-k+1} \ldots \alpha_{N} z_{N} \in \sum_{i=0}^{k-2} \bigcap_{U_{N-k+i} Q_{N-k+i}} \\
& {\left[-\bar{\gamma}_{i} \mathrm{M}_{1}+\prod \alpha_{N-k} \ldots \alpha_{N-k+i} \beta_{N-k+i+1}\left(P_{N-k+i}, v_{N-k+i}\right)\right]} \\
& +\prod \alpha_{N-k} \ldots \alpha_{N-1} \beta_{N}\left(\bar{u}_{N-1}, \bar{v}_{N-1}\right)-\bar{\gamma}_{k-1} m_{1, k-1 .} .
\end{aligned}
$$

It is equivalent to the following

$-\prod \alpha_{N-k} \alpha_{N-k+1} \ldots \alpha_{N-1}\left[\alpha_{N} z_{N}+\beta_{N}\left(\bar{u}_{N-1}, \bar{v}_{N-1}\right)\right] \in \bar{\gamma}_{k-1} m_{1, k-1}$

$+\sum_{i=0}^{k-2} \bigcap_{N_{N-k+i} \in Q_{N-k+i}}\left[-\bar{\gamma}_{i} \mathrm{M}_{1}+\prod \alpha_{N-k} \ldots \alpha_{N-k+i} \beta_{N-k+i+1}\left(P_{N-k+i}, v_{N-k+i}\right)\right.$

Therefore owing to (32) we have

$$
\begin{aligned}
& -\prod_{k=2} \alpha_{N-k} \alpha_{N-k+1} \ldots \alpha_{N-1} z_{N-1} \in \bar{\gamma}_{k-1} m_{1, k-1} \\
& +\sum_{i=0} \bigcap_{N-k+i \in Q_{N-k+i}}\left[-\bar{\gamma}_{i} \mathrm{M}_{1}+\prod \alpha_{N-k} \ldots \alpha_{N-k+i} \beta_{N-k+i+1}\left(P_{N-k+i} U_{N-k+i}\right)\right] .
\end{aligned}
$$

In the same way, if the control $\bar{v}_{N-2}$ becomes the stated way known that we above us can construct controls $\bar{u}_{N-2}$ providing inclusion

$$
\begin{aligned}
& -\prod \alpha_{N-k} \alpha_{N-k+1} \ldots \alpha_{N-2} z_{N-2} \in \bar{\gamma}_{k-1} m_{k-1}+\bar{\gamma}_{k-2} m_{k-2} \sum_{i=0}^{k-3} \bigcap_{N_{N-k+i} \in Q_{N-k+i}} \\
& {\left[-\bar{\gamma}_{i} \mathrm{M}_{1}+\prod \alpha_{N-k} \ldots \alpha_{N-k+i} \beta_{N-k+i+1}\left(P_{N-k+i}, v_{N-k+i}\right)\right]}
\end{aligned}
$$

etc. Thus, we will receive

$$
\begin{aligned}
& -\prod \alpha_{N-k} \alpha_{N-k+1} \ldots \alpha_{N-2} z_{N-2} \in \bar{\gamma}_{k-1} m_{k-1}+\bar{\gamma}_{k-2} m_{k-2} \sum_{i=0}^{k-3} \bigcap_{U_{N-k+i} \in Q_{N-k+i}} \\
& {\left[-\bar{\gamma}_{i} \mathrm{M}_{1}+\prod \alpha_{N-k} \ldots \alpha_{N-k+i} \beta_{N-k+i+1}\left(P_{N-k+i}, v_{N-k+i}\right]\right.}
\end{aligned}
$$

from here we receive

$$
z_{N-k-1} \in \mathrm{M} \text {. }
$$

The theorem is proved completely.

Proof of Theorem 4. Let in game (13) one be able to complete the pursuit from "boundary" situation $\left(f_{0}, f_{N}\right)$ $=\left(-y_{0},-y_{N}\right)$ in $N$ steps. Then, it follows from Definition 2 that from any sequence $\bar{v}_{0}, \bar{\nu}_{1}, \ldots, \bar{v}_{N-1}, \bar{v}_{k} \in Q$, $0 \leq k \leq N-1$, of the evasion control it is possible to construct a sequence $\bar{u}_{0}, \bar{u}_{1}, \ldots, \bar{u}_{N-1}, \bar{u}_{k} \in P, \quad 0 \leq k \leq N-1$, of pursuit control such that the solution $\left(z_{0}, \bar{z}_{1}, \ldots, z_{N-1}, z_{N}\right)$ of the equation $z_{1}=X_{0} z_{0}+y_{0}, \quad z_{n+1}+A_{n} z_{n}-z_{n-1}=F_{n}, 1 \leq n$ $\leq N-1, \quad z_{N-1}=X_{1} z_{N}+y_{N}$, for some $d \leq N$ hits $M: \bar{z}_{d}$ $\in M$. Let now in game (2) $v=\bar{v}(x, y) \in \bar{Q},(x, y) \in \Omega$, be an arbitrary control of an evader from the class $L_{2}(\Omega)$. With the knowledge of the evader control $v=\bar{v}(x, y)$, it is possible to determine $\bar{v}_{i, k}$ as the values of this function at the node points of the grid $\Omega_{h l}$, that is,

$$
v_{k}=\bar{v}_{k}=\left(\bar{v}_{1, k}, \bar{v}_{2, k}, \ldots, \bar{v}_{r-1, k}\right) .
$$

Whence it follows that in virtue of Theorem 4 we can construct the pursuer control in game (13) providing completion of pursuit

$$
u_{k}=\bar{u}_{k}=\left(\bar{u}_{1, k}, \bar{u}_{2, k}, \ldots, \bar{u}_{r-1, k}\right) .
$$

Now in game (2) we construct the pursuer control $u=\bar{u}(x, y)$ as follows: $\bar{u}(x, y)=\left\{\bar{u}_{i, k}=\bar{u}_{i, k}: i h \leq x_{i}<<(i+1) h\right.$, $i=0,1, \ldots, r-1, k l \leq y<(k+1) l, k=0,1, \ldots, \theta-1\}$.

Obviously, $u \in P$ and $\bar{u}(x, y) \in L_{2}(\Omega)$. By substituting $v=\bar{v}(x, y)$ and $u=\bar{u}(x, y)$ in (2), we obtain a differen- 
tial equation. Similarly, by substituting $\bar{v}_{i, k}$ and $\bar{u}_{i, k}$ in (3), we obtain a grid equation approximating equation (2).

Let $(\bar{z})_{h l}$ be the value of the exact solution corresponding to the controls $v=\bar{v}(x, y)$ and $u=\bar{u}(x, y)$ of problem (2) at the nodes of the grid $\Omega_{h l}, \bar{z}_{i, k}$ be the solution corresponding to the controls $\bar{v}_{i, k}$ and $\bar{u}_{i, k}$ of the difference problem (3). Then, we obtain from (13) and the condition of Theorem 4 that

$$
\left\|(z)_{h l}-\bar{z}_{i, k}\right\|_{\Phi_{h l}} \leq K_{1} l+K_{2} h^{2}<\varepsilon .
$$

From this fact and $\bar{z}_{i, k} \in \bar{M}_{1}$, we obtain $(z)_{h l}-\bar{z}_{i, k} \in \varepsilon S$, $(z)_{h l} \in \varepsilon S+\bar{z}_{i, k},(z)_{h l} \in \varepsilon S+\bar{M}_{1}$, which proves the theorem.

\section{Conclusions}

Thus, to solve the game problem of pursuit in the form (1) we pass to the discrete game (13) or (14), and Theorems 1-3 establish the sufficient condition for such problems. Theorem 4 establishes the sufficient conditions for solving the problem of pursuit (1). Here, the difference $(z)_{h l}-z_{i, j} \quad$ (see Section 3) plays the main part in the solution of problem and implies that the solutions of the grid equation (2) are stable.

The problem of stability of the grid equation (2) lies in determining the conditions under which the numerical error $p_{i, j} \equiv(z)_{h l}-z_{i, j}$ tends to zero with growing $j$ uniformly in all $i, 0 \leq i \leq n$, or at least remains bounded.

Equation (2) is called stable if the round off errors generated in the course of calculations have tendency to decrease or at least not to increase. Otherwise, the accumulated errors may reach a value such that the numerical solution $(z)_{h l}$ has nothing in common with the exact solution of the grid problem (2). It goes without saying that such unstable grid equations cannot be used for numerical solution of the differential games.

Theorems 1-4 are easily generalized to a wider class of differential games, for example, when

$$
\sum_{\alpha=1}^{n} a_{\alpha}\left(x_{1}, x_{2}, \ldots, x_{n}\right) \frac{\partial^{2} z}{\partial x_{\alpha}^{2}}=f\left(u\left(x_{1}, x_{2}, \ldots, x_{n}\right), v\left(x_{1}, x_{2}, \ldots, x_{n}\right)\right)
$$

with discontinuous coefficients.

\section{REFERENCES}

[1] O. A. Ladyzhenskaya, "Kraevye Zadachi Matematicheskoi Fiziki,” (Boundary Problems of Mathematical Physics), Moscow, Nauka, 1973.
[2] O. A. Ladyzhenskaya, V. A. Solonnikov and N. N. Ural'tseva, "Lineinye I Kvazilineinye Uravneniya Parabolicheskogo Tipa,” (Linear and Quasi linear Functions of Parabolic Type), Moscow, Nauka, 1967.

[3] V. A. Il'in, "Boundary Control of String Oscillations at One End with Other End Fixed, Provided that Finite Energy Exists,” Dokl. Ross. Akad. Nauk, Vol. 378, No. 6, 2001, pp. 743-747.

[4] V. A. Il'in and V. V. Tikhomirov, "Wave Equation with Boundary Control at Two Ends and Problem of Complate Oscillation Damping ,” Diff. Uravn., Vol. 35, No. 5, 1999, pp. 692-704.

[5] Yu. S. Osipov and S. P. Okhezin, "On the Theory of Differential Games in Parabolic Systems,” Dokl. Akad. Nauk SSSR, Vol. 226, No. 6, 1976, pp. 1267-1270.

[6] F. L. Chernous'ko, "Bounded Controls in Distributed-parameter Systems,” Prikl. Mat. Mekh., Vol. 56, No. 5, 1992, pp. 810-826.

[7] N. Satimov and M. Sh. Mamatov, "On a Class of Linear Differential and Discrete Games between Groups of Pursuers and Evaders,” Diff. Uravn., Vol. 26, No. 9, 1990, pp. 1541-1551.

[8] N. Satimov and M. Tukhtasinov, "On some Game Problems in the Distributed Controlled Systems,” Prikl. Mat. Mekh., Vol. 69, No. 6, 2005, pp. 997-1003.

[9] N. Satimov and M. Tukhtasinov, "On some Game Problems in Controlled First-order Evolutionary Equations," Diff. Uravn., Vol. 41, No. 8, 2005, pp. 1114 -1121.

[10] M. Sh. Mamatov, "On the Theory of Differential Pursuit Games in Distributed Parameter Systems,” Automatic Control and Computer Sciences, Vol. 43, No. 1, 2009, pp. 1-8. doi:10.3103/S0146411609010015

[11] M. Sh. Mamatov, "About Application of a Method of Final Differences to the Decision a Prosecution Problem in Systems with the Distributed Parameters," Automation and Remote Control, Vol. 70, No. 8, 2009, pp. 1376-1384. doi:10.1134/S0005117909080104

[12] M. Tukhtasinov and M. Sh. Mamatov, "On Pursuit Problems in Controlled Distributed Systems," Mathematical notes, Vol. 84, No. 2, 2008, pp. 273-280.

[13] M. Tukhtasinov and M. Sh. Mamatov, "About Transition Problems in Operated Systems," Diff. Uravn., Vol. 45, No.3, 2009, pp. 1-6.

[14] M. Sh. Mamatov and M. Tukhtasinov, "Pursuit Problem in Distributed Control Systems," Cybernetics and Systems Analysis, Vol. 45, No. 2, 2009, pp. 297-302. doi:10.1007/s10559-009-9100-X

[15] G. I. Marchuk, “Metody Vychislitel'noi MateMatiki,” (Methods of computational Mathematics), Moscow, Nauka, 1989. 